leur rapport $\mathrm{Ca} / \mathrm{P}$ est du même ordre de grandeur que celui des petits-suisses.

On voit que certains fromages frais peuvent contribuer, pour une large part, à la couverture des besoins calciques de notre organisme. Ainsi, pour remplacer, à ce point de vue, $1 / 4$ de litre de lait, il suffirait de consommer 80 grammes d'un fromage blanc très riche en calcium, tel que celui que nous avons préparé nous-mêmes au laboratoire avec de la présure.

(Travail du Laboratoire de Physiologie de la Nutrition du Centre National de la Recherche Scientifique, et de l'Institut National de la Recherche agronomique).

\title{
BIBLIOGRAPHIE
}

[1] L. Randorn et C. VAtinel. Le Lait, 1951, 31, 113.

[2] L. Randoin et C. Jourdan-Vatinel. Le Lait, 1951, 31, 250.

[3] G. Guittonneau et R. Chevalier. C. R. Ac. Sc., 1934, 199, 801.

[4] Ch. O. Guillaumin. Bull. Soc. Ohim. biol., 1932, 14, 105.

[5] H. Copaux. C. R. Ac. Sc., 1921, 173, 656.

[6] L. Randoin, P. Le Gallic et J. Causeret. Tables de composition des aliments ( $2^{e}$ édition), précédées des Normes de l'alimentation humaine. Lanore, éditeur. Paris, 1947.

\section{SUR LA FORMATION DE L'AROME DU BEURRE par}

\section{A. C. F. KRUIJER}

Bien que le dernier mot n'ait pas été dit en ce domaine et qu'il $\mathrm{y}$ ait des points de vue complémentaires à attendre, il est peut-être justifiable de résumer la théorie de Van Beynum ét Peтte, extraite de leur livre "Microbiologie laitière et hygiène du lait ", écrit en collaboration avec SJollema (1).

Cette théorie se résume ainsi : le lait contient jusqu'à 2 grammes d'acide citrique $\left(\mathrm{C}^{6} \mathrm{H}^{8} \mathrm{O}^{7}\right.$. $\left.\mathrm{H}^{2} \mathrm{O}\right)$ par litre, décomposé par l'action conjuguée du Betacoccus cremoris et des streptocoques Lactis et Cremoris; les facteurs qui dirigent cette décomposition sont le degré d'acidité du milieu et la présence ou l'absence d'oxygène. Indépendamment de l'acidité et de l'oxygène, les deux premières étapes s'exécutent ainsi :

(1) J. Van Beynum, J. W. Pette et P. Sjollema. Zuivelmicrobiologie en Melkhygiene. Editeur : C. Misset N. V. - Doetinchen, Hollande, 1950. 
I<smiles>O=CC(CC(=O)C(C(=O)O)C(=O)O)C(O)C(=O)O</smiles>

acide citrique acide acétique acide pyruvique II

$$
\begin{aligned}
& \mathrm{CH}^{3} \\
& \mathrm{COOH}
\end{aligned} \rightarrow \mathrm{CO}^{2}+\underset{\mathrm{H}-\mathrm{C}=\mathrm{O}}{\mathrm{CH}^{3}} \text { acétaldéhyde }
$$

Pour la troisième étape, on peut classer les possibilités de la manière suivante :

III-A : en lait neutre

$$
\begin{aligned}
& \text { III-B : en lait acide } \rightarrow \text { III-B-a : sans oxygène } \\
& \text { III-B-b : avec oxygène }
\end{aligned}
$$

III-A : en lait neutre

$$
\begin{aligned}
& 2 \underset{\mathrm{H}-\mathrm{C}=\mathrm{O}}{\mathrm{CH}^{3}}+\mathrm{H}^{2} \mathrm{O} \rightarrow{\underset{\mathrm{COOH}}{\mathrm{CH}^{3}}}_{\mathrm{H}^{2}-\mathrm{C}-\mathrm{OH}}^{\mathrm{CH}^{3}} \\
& \text { aethanol }
\end{aligned}
$$

III-B-a : en lait acide, sans oxygène

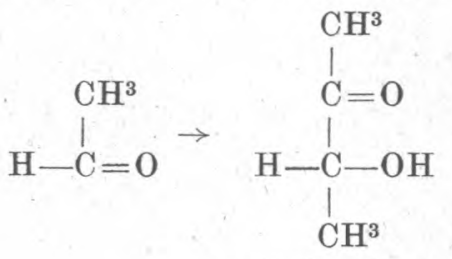

acétyl-méthyl-carbinol

III-B-b : en lait acide, avec oxygène

Formation du carbinol suivant III-B-a, et en plus, du diacétyle

$$
2 \quad \begin{aligned}
& \mathrm{CH}^{3} \\
& \mathrm{H}-\mathrm{C}=\mathrm{O}
\end{aligned}
$$


Donc, en résumé : 2 molécules d'acide citrique donnent :

$1^{\circ}$ en milieu neutre, $4 \mathrm{~mol}$. de dioxyde de carbone $+3 \mathrm{~mol}$. d'acide acétique +1 mol. d'aethanol ;

$2^{\circ}$ en milieu acide, $4 \mathrm{~mol}$. $\mathrm{CO}^{2}+2$ mol. d'acide acétique + 1 mol. de " produit $-\mathrm{C}^{4}$ ).

Les facteurs nécessaires à la formation du diacétyle sont par conséquent :

de l'acide citrique (citrates du lait);

un milieu acide (acide lactique) ;

del'oxygène (l'atmosphère) ;

des bactéries Sc. Lactis et Cremoris et Bc. Cremoris (flore du lait).

L'odeur typiquement aromatique d'une culture acide servant à la maturation de la crème pour le beurre, est due au mélange de diacétyle, d'acice acétique et d'acide carbonique (une partie de cet acide carbonique se décompose, surtout dans la baratte, et le dioxyde de carbone se dégage).

La réduction des "produits $-\mathrm{C}^{4}$ » donne finalement le 2-3 butylène-glycol, autre produit- $\mathrm{C}^{4}$ :<smiles>CCC(=O)C(O)CC(=O)C=CC(=O)C(C)O</smiles>

2-3 butylène-glycol

Cette dernière réaction dépend du caractère des ferments producteurs d'arome (les uns réduisent plus fortement que les autres) et du degré d'acidité (plus ce degré est élevé, plus la réduction est poussée).

Le pourcentage des produits- $\mathrm{C}^{4}$ sous forme de glycol s'appelle "pourcentage-réduction $\mathbf{R}$ ". $\mathbf{R}$ croît avec le temps; si $\mathbf{R}>50$ au moment où tout l'acide citrique est décomposé, le mélange est trop réducteur.

Afin d'en tirer des eonclusions pratiques pour la maturation de la crème en vue de la fabrication du beurre, on peut retenir les points suivants :

1. La culture ne doit pas contenir de microorganismes autres que Sc. Lactis Sc. Cremoris (formant du d-acide lactique) et Bc. Cremoris (caractéristiques du Betacoccus : par paires ou en 
chaînes, Gram +, catalase - , pas de spores, i mmobile, formant les bases de l'arome et un peu de 1-acide lactique).

2. L'équilibre entre les bactéries Sc. Lactis et S. Cremoris, et $B c$. Cremoris doit être tel que la décomposition de l'acide citrique par les Bc. Cremoris ait lieu au bon moment $(\mathrm{R}<50)$ à un haut degré d'acidité.

3. Les Bc. Cremoris doivent être de caractère faiblement réducteur.

4. Les spécificités de la culture acide doivent être maintenues pendant et après l'ensemencement.

Pour contrôler la force réductrice du mélange, on peut se servir de la réaction de la créatine, qui accuse une couleur rouge en présence du carbinol. Bien que cette couleur ne donne pas une mesure de la quantité de diacétyle formé, elle nous orientera sur la formation de l'arome dans une culture acide gardée pendant vingt. quatre heures.

Le glycol ne colore pas la créatine; une bonne culture doit avoir une réaction positive envers la créatine pendant trois jours (à une température de 18 à $20^{\circ}$ C.).

Manipulation de ce contrôle : dans un tube à essai on ajoute à $2 \mathrm{~cm}^{3} 5$ de culture acide $1 \mathrm{~cm}^{3} \mathrm{~d}^{\prime}$ une solution contenant 1 gramme de créatine pour $100 \mathrm{~cm}^{3}$ d'eau distillée, et $2 \mathrm{em}^{3} 5$ d'hydroxyde de sodium (contenant 40 grammes $\mathrm{NaOH}$ pour $100 \mathrm{~cm}^{3}$ d'eau); on ferme le tube avec un bouchon en caoutchouc. On agite fortement, en répétant cette agitation plusieurs fois dans le courant de la première demi-heure, après quoi on juge l'intensité de la couleur rouge.

Terminons en remarquant que dans le beurre frais on ne trouve que $1 \mathrm{mgr}$. 0 de diacétyle par kilogramme, cette quantité augmentant jusqu'aux environs de $1,7 \mathrm{mgr} / \mathrm{kg}$. ("arome») et de $2,6 \mathrm{mgr} . / \mathrm{kg}$. (arome fort) après stockage pendant une semaine à une température de $14^{\circ} \mathrm{C}$.

\section{BIBLIOGRAPIIIE ANALYTIQLE}

\section{LES LIVRES}

Davis (J. G.). - Milk testing. The laboratory control of milk (Analyse du lait. Contrôle du lait au laboratoire). 1 livre illustré relié toile de 260 pages. Dairy Industries, Ltd., 24, Bride Lane, London E. C. 4, 1951. Prix : 15 shillings.

Le $D^{r} J$. G. Davis, auteur de l'excellent livre que nous présentons aujourd'hui à nos lecteurs, est Directeur technique d'une des plus grandes sociétés 